\title{
Effect of Water Depth Change on Manning Coefficient for Partially-Filled Circular Culverts
}

تأثير تغيير أعماق المياه على معامل ماننج للبر ابخ الدائرية المملو عة جزئيا

\section{Marwa EL-Sayed A. Meky ${ }^{1}$, Shimaa M. Ghoraba ${ }^{2}$, I.M.H. Rashwan ${ }^{3}$}

${ }^{1}$ Demonstrator, Civil Engineering Department, Higher Institute of Engineering and Technology, Kafr El-Sheikh, Egypt,

${ }^{2}$ Lecturers, Irrigation and Hydraulics Engineering Department, Faculty of Engineering-Tanta University, Egypt,

${ }^{3}$ Professor, Head of Irrigation and Hydraulics Engineering Department, Faculty of Engineering, Tanta University, Egypt, imh_rashwan@yahoo.com, 01226573674

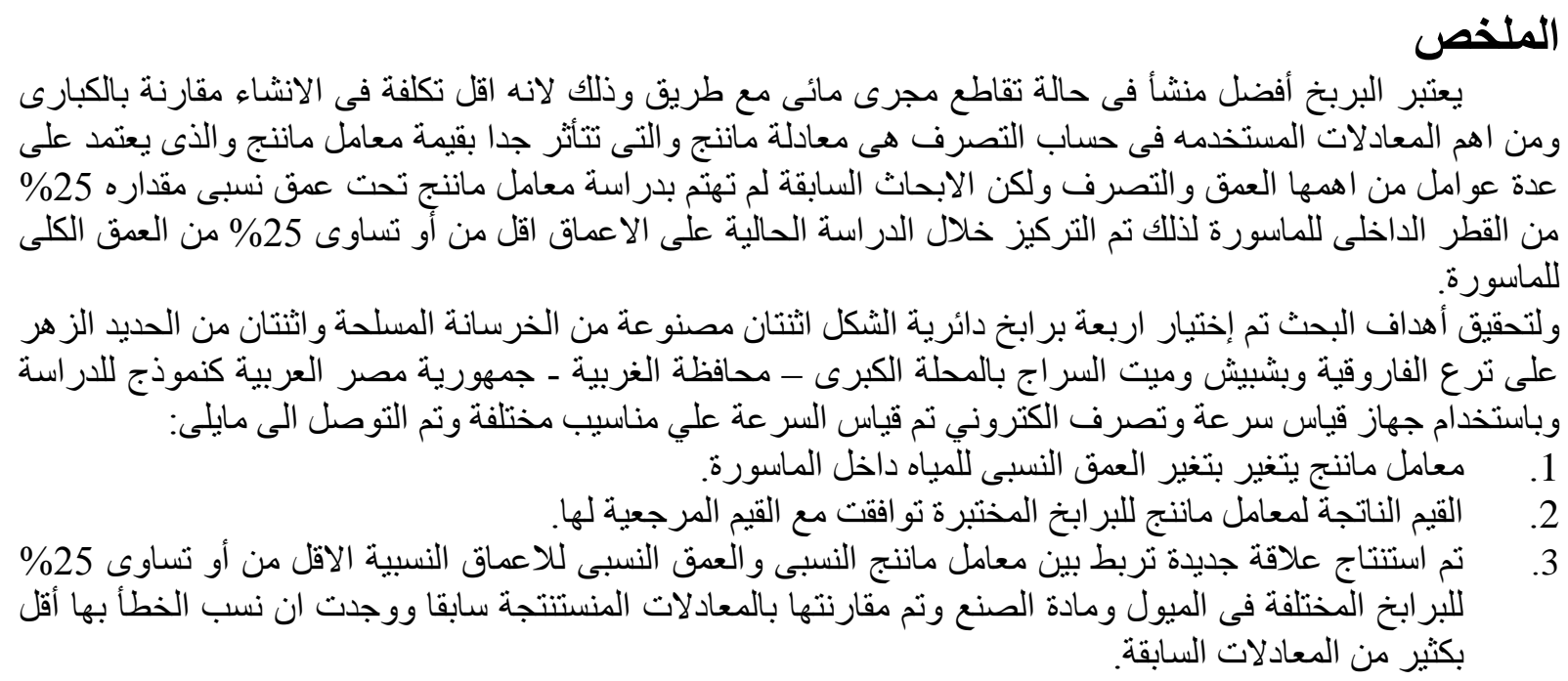

\begin{abstract}
Culvert is a structure used to convey surface runoff through embankments. The circular is the popular section for design pipes, such as culverts, drain tiles and sewers of small and medium sizes. Also it may be made of concrete, steel, corrugated metal and polyethylene. Manning's equation is most commonly used throughout the world for the purpose of computing flow velocities in open channel. Manning roughness coefficient is one of the effective factors on water velocity and discharge. The present study is about the determination of the relation between relative depth and relative roughness for circular culvert. For this purpose, field work was carried out on four pipe culverts on canals at El-Mahalla El kubra, Egypt. The culverts are of different diameters, slopes and materials. The velocity is recorded by using FlowTracker. Manning roughness coefficient greatly varied with the variation of relative depth, surface roughness and discharge. For four culverts, a total of 143 depth data points were collected at different discharges ranging from 0.004 to $4.750 \mathrm{~m}^{3} / \mathrm{sec}$. New equations are developed for relation between the relative depth $(d / D)$ and the relative roughness $\left(n / n_{f}\right)$ for flow depths less than $25 \%$ full for four pipe culverts on El Mahalla El Kubra, Egypt, when the water geometries are given.
\end{abstract}

\section{Keywords:}

Manning's coefficient, Manning's equation, open channel, culvert, pipe, relative depth, and relative roughness. 


\section{Introduction}

Manning's equation was introduced by the Irish Engineer Robert Manning in 1889, Chow [1]. It is an empirical equation that applies to uniform flow in open channels and is a function of the channel velocity, flow area and channel slope. Manning's equation formula is:

$$
V=\frac{1}{n} R^{\frac{2}{3}} S^{\frac{1}{2}}
$$

Where $V=$ mean velocity, $n=$ the Manning's roughness coefficient, $R=$ the hydraulic radius and $S=$ the bed slope.

Major factors affecting Manning's roughness coefficient are, surface roughness, vegetation, channel irregularity, channel alignment, silting and scouring, obstruction, size and shape of channel, stage and discharge, seasonal change, suspended material and bed load.

Yarnell and Woodward [2] carried out their research on clay and concrete drain tile with diameter from 4 to 12 inches with slopes from 0.05 to $1.5 \%$. Their study was not based on Manning's roughness $n$ but on the Kutter $n$ and the Chezy $C$ coefficient. Camp [3] developed the partial full flow investigations of Wilcox and Yarnell and Woodward with research investigation circular sewer pipe designed to flow partially full. He noted that the researches of Yarnell and Woodward include data indicating Manning's roughness values at pipes under partial flow greater than at full flow. Camp's work led to the curve, which shows the variation of $Q / Q_{\text {full }}, V / V_{\text {full }}$, and $n / n_{\text {full }}$ as functions of the ratio of depth of flow to pipe diameter $(y / D) . V_{\text {full }}$ and $Q_{\text {full }}$ can be calculated for full pipe flow conditions in a given pipe with the Manning equation. In 1951, Straub and Morris [4] carried out a research on corrugated circular and the pipe arch culverts. In circular case they applied their application on diameter pipe sections were 18, 24 and 36 inch with $193 \mathrm{ft}$ long and lay on a slope of $0.2 \%$. They noted that Manning's roughness coefficient decreased as diameters increased for a relative depth. From Straub and Morris curve's it was noticed that decreasing relative depth does not vary with relative roughness values. In 1954, Cosens [5] carried out a research on asbestos cement and vitrified clay with diameter 8 inch and slopes of 0.25 and $0.4 \%$. He noted that the velocity at $50 \%$ capacity and full flow was not equal. He also noted that the slope of the energy grade line increases the roughness increasing. In 1964, Neale and Price [6] carried out their research on polyvinyl chloride pipe (PVC) with diameter 8 and 12 inch and slopes of $0.3,0.6$ and $1 \%$ under both partially full and full flow. From their curve it is clear that relative depth decreases while the relative roughness remains approximately constant and independent of the velocity. In 1967, Pomeroy [7] confirmed that the $n$ value is a coefficient in an empirical equation and it is defined as a mathematical function of the variables of the equation. $\mathrm{He}$ calculated Manning's roughness for the individual depth measurements and took the average ignoring the data points for which the Froude numbers were between 0.9 and 1.4. He collected the data in 21 existing sewer lines. The total number of data points collected by him below $20 \%$ relative depth was 78 and the maximum relative depth attained during his experiment was $69.9 \%$. He did not end up calculating full flow Manning's roughness for the sewers because full flow was never attained in those sewer lines.

In 1986, Barr and Das [8] proposed two explicit equations for $h / D$ but did not indicate how velocity $V$ showed be calculated. Saatçı $(1990,1992)[9,10]$ proposed another two explicit solutions for $\theta$ and calculated the flow depth $h / D$ and velocity $V$. In 1993 Esen [11] proposed new two explicit solutions calculated the flow depth $h / D$ and velocity $V$. In $1994 \mathrm{Li}$ [12] proposed two explicit solutions for $\theta$ and calculated the slope $S$. 1998, Zaghloul [13] showed that the roughness value $n$ for a pipe partially full 
is greater than the roughness value $n$ for full flow conditions. Giroud et al. (2000) [14] proposed direct estimation of $V$ (without calculating $\theta$ or $h / D$ first). Akgiray (2004) [15] reviewed types of problems that require iterative calculations and explicit solutions proposed in the literature as:

I . Given $Q, D$, and $S$, find, $h / D$ and (or) $V$; and

II .Given $Q, D$, and $V$, find, $h / D$ and (or) $S$ He reexamined type I and type II problems and found that Camp's curve was well represented by a new and simpler alternative equation. $\mathrm{He}$ developed a single relation to replace the two Saatçı equations given before him. Akgiray (2005) [16] improved new equations for two other types of problem as:

- Given $V, D$, and $S$, find, $d / D$ and (or) $Q$; and

- $\quad$ Given $Q, V$, and $S$, find, $d / D$ and (or) $D$.

In 2009, From American Concrete Pipe Association [17] presented a method for determination the values of the partial flow depth and velocity in circular concrete pipe. In 2010, Mangin [18] used the previous data to develop an equation to predict water depth inside of partially circular culverts independently from the Manning's equation. The new equation predicts depth more frequently and with greater accuracy than the Manning's equation for the data analyzed and is therefore, a more consistent method when used to design and assess culverts for fish passage. This equation to predict depth $(d)$ for a circular culvert, based on discharge $(Q)$, slope $(S)$, diameter $(D)$, gravity $(g)$ and absolute material roughness $(K s)$, has been developed. The new equation reduces the absolute mean error in calculating water depth by $37 \%$ compared to the previously published data. In 2012, Devkota [19] said that previous researchers have found that the Manning's roughness for partial flows is greater than full flow but inadequate data were collected for flows less than $20 \%$ full. So he tried to collect water depth data in HDPE culverts and to derive a relation between Manning's roughness and relative depth with a $4 \mathrm{ft} \times 2 \mathrm{ft}$ flume of $60 \mathrm{ft}$ length, three test culverts of diameter $1 \mathrm{ft}, 2 \mathrm{ft}$ and $3.5 \mathrm{ft}$ were tested with the discharge ranging from 0.2 to $10.3 \mathrm{cfs}$ at bed slopes of 0.2 to $2 \%$. He showed that the Manning's roughness coefficient varied with diameter, relative depth, slope and discharge. Also he showed that with the increase in the slope of energy grade line, the Manning's roughness coefficient also increases. Devkota [19] found that between $20 \%$ and $40 \%$ relative depth the partial flow roughness was found to be roughly equal to the full flow roughness. The results further indicate that the peak Manning's roughness coefficient for partial flow in the 1,2 and 3.5ft diameter culvert was 0.011 and occurred at about $27.5 \%$ of the full flow. The fact that below $20 \%$ depth, the Manning's roughness coefficient is smaller than the design roughness (full flow roughness) causes the flow velocity to be higher than predicted or designed. Similarly from 20 to $40 \%$ full, the culvert has higher roughness indicating lower velocity than otherwise would be predicted.

In 2012, Toews and Clark [20] carried out their research on CMP (corrugated metal pipe) culvert with diameter $0.8 \mathrm{~m}$ of $21 \mathrm{~m}$ long and the culvert was supported on a series of adjustable yokes to allow slope adjustment. They measured relative depths ranged from 0.03 to 0.67 for each of the tested slopes $(0.04,0.14,0.27,0.49$ and $0.75 \%$ ). Therefore a set of equations were developed, using a least squares regression for the variation in Manning's $\mathrm{n}$. They noted that their equations did not give satisfactory results for the flow depth below relative depth of 0.2 so that they used Mangin's dimensionless parameters $S$ and $Q / \sqrt{g D^{5}}$. In 2012, Bengtson [21] gave an equation for relative roughness value $n / n_{f}$ for a pipe partially full. 
The goal of this study is to investigate the effect of variation of water depth in types of culvert materials available and to determination a relation between Manning's roughness coefficient and relative depth. The water depth profile was measured within culverts with varying discharge, slope and diameter. Discharge and depth thus collected were used to calculate Manning's roughness coefficient. The coefficients were then compared to the full flow roughness reported for steel and concrete culverts by Chow [1].

\section{Field Work}

Manning's roughness coefficient in culvert changes with water depth, discharge, diameter, slope and culvert's surface roughness. The field work was used to conduct experiments that would determine the variation of Manning's roughness coefficient with relative depth. Concrete and steel culverts were studied because it is the most common types of culvert materials. Most of the previous investigators did not use the steel culvert in their researches. Culverts under consideration are constructed at ELFarokya, Beshbesh and Meet EL-Serag canals. The tested culverts are circular. The diameters of the test culverts were 1.0, 1.4, $1.7,2.0 \mathrm{~m}$ with four different slopes 0.001 , $0.003,0.0017$ and 0.00125 respectively.

The measurements quantities were discharge and water depth in the culvert. Upstream the canal intake, control gate was installed to limit the discharge of water into the culvert. The tested culverts are defined with each location $\mathrm{km}$ on canal, material, diameter, length, upstream and downstream bed level and slope as shown in Table (1).
Table (1) Illustration of Bed Slopes and Locations of Culverts

\begin{tabular}{|c|c|c|c|c|}
\hline Culvert Site & 1 & 2 & 3 & 4 \\
\hline Canal Name & $\begin{array}{c}\text { EL- } \\
\text { Farokya }\end{array}$ & Beshbesh & $\begin{array}{c}\text { Met } \\
\text { EL- } \\
\text { Serag }\end{array}$ & $\begin{array}{c}\text { Met } \\
\text { EL- } \\
\text { Serag }\end{array}$ \\
\hline Km & 2.400 & 3.250 & 6.750 & 5.000 \\
\hline Material & Concrete & Concrete & Steel & Steel \\
\hline Diameter (m) & 1.0 & 1.4 & 1.7 & 2.0 \\
\hline Length (m) & 10 & 6 & 6 & 8 \\
\hline $\begin{array}{c}\text { Upstream Bed } \\
\text { Level }\end{array}$ & $(2.51)$ & $(1.82)$ & $(2.16)$ & $(2.27)$ \\
\hline $\begin{array}{c}\text { Downstream } \\
\text { Bed Level }\end{array}$ & $(2.50)$ & $(1.80)$ & $(2.15)$ & $(2.26)$ \\
\hline Slope & 0.001 & 0.003 & 0.0017 & 0.00125 \\
\hline
\end{tabular}

\subsection{Experimental Measurements}

Field data have been collected at four different sites. For the first culvert, thirty-one sections were chosen, for the second culvert, thirty-nine sections were chosen.

As, for third culvert, forty-one sections were chosen and thirty-three sections were chosen for fourth one. The field measurements were water depths and velocities.

\subsubsection{Water depth}

Water surface varied with the control discharge gate. For each discharge, water depth was measured.

For these experiments, water depth in the culvert was measured by scalar rod. Measurements are taken when the situation is steady and the water levels are stable.

\subsubsection{Mean Velocity}

The culvert cross section was divided into sections, as shown in Fig. (1).

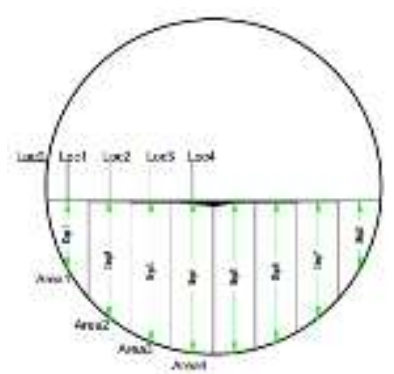

Figure (1) Divisions the Culvert Cross Section into Station

In the present study, the mean sectional station velocity was determined by measuring the velocity at 0.6 of the depth 
in each vertical $\left(V_{\text {mean }}=V_{0.6}\right)$, or, where more reliable results were required, by taking the average of the velocities at 0.2 and 0.8 the depth $\left(V_{\text {mean }}=\left(V_{0.2}+V_{0.8}\right) / 2\right)$.

For the culvert cross section, series of velocity measurements were taken at different locations for each water depth using the FlowTracker. The mean velocity was determined at that station for more depths.

\subsubsection{Experimental Results}

Field data have been collected at four different culverts sites.

All measurements are tabulated in Tables (2) to (5)

Table (2) Measurements of Water Depths and Mean Velocities for Concrete Culvert

\begin{tabular}{|c|c|c|c|c|c|c|c|}
\hline \multicolumn{4}{|c|}{$D=1.0 \mathrm{~m}, Q_{f}=2.053 \mathrm{~m}^{3} / \mathrm{s}$ and $S=0.001$} & \multicolumn{5}{c|}{$D=1.0 \mathrm{~m}, Q_{f}=2.053 \mathrm{~m}^{3} / \mathrm{s}$ and $S=0.001$} \\
\hline Run No. & $d(\mathrm{~m})$ & $d / D$ & $V_{\text {mean }}(\mathrm{m} / \mathrm{s})$ & Run No. & $d(\mathrm{~m})$ & \multicolumn{2}{c|}{$V_{\text {mean }}(\mathrm{m} / \mathrm{s})$} \\
\hline 1 & 0.05 & 0.05 & 0.2997 & 17 & 0.50 & 0.50 & 0.8313 \\
\hline 2 & 0.10 & 0.10 & 0.4281 & 18 & 0.53 & 0.53 & 0.8553 \\
\hline 3 & 0.12 & 0.12 & 0.4665 & 19 & 0.55 & 0.55 & 0.8685 \\
\hline 4 & 0.15 & 0.15 & 0.5262 & 20 & 0.57 & 0.57 & 0.8628 \\
\hline 5 & 0.20 & 0.20 & 0.5865 & 21 & 0.60 & 0.60 & 0.8748 \\
\hline 6 & 0.22 & 0.22 & 0.6111 & 22 & 0.63 & 0.63 & 0.9147 \\
\hline 7 & 0.25 & 0.25 & 0.6438 & 23 & 0.65 & 0.65 & 0.8904 \\
\hline 8 & 0.28 & 0.28 & 0.6639 & 24 & 0.67 & 0.67 & 0.9114 \\
\hline 9 & 0.30 & 0.30 & 0.6903 & 25 & 0.70 & 0.70 & 0.9057 \\
\hline 10 & 0.33 & 0.33 & 0.7218 & 26 & 0.74 & 0.74 & 0.9429 \\
\hline 11 & 0.35 & 0.35 & 0.7227 & 27 & 0.76 & 0.76 & 0.9282 \\
\hline 12 & 0.37 & 0.37 & 0.7479 & 28 & 0.80 & 0.80 & 0.9789 \\
\hline 13 & 0.40 & 0.40 & 0.7524 & 29 & 0.86 & 0.86 & 0.9915 \\
\hline 14 & 0.42 & 0.42 & 0.7503 & 30 & 0.90 & 0.90 & 1.0338 \\
\hline 15 & 0.45 & 0.45 & 0.7824 & 31 & 0.97 & 0.97 & 1.0533 \\
\hline
\end{tabular}

Table (3) Measurements of Water Depths and Mean Velocities for Concrete Culvert

\begin{tabular}{|c|c|c|c|c|c|c|c|}
\hline \multicolumn{4}{|c|}{$D=1.4 \mathrm{~m}, Q_{f}=0.82 \mathrm{~m}^{3} / \mathrm{s}$ and $S=0.003$} & \multicolumn{4}{|c|}{$D=1.4 \mathrm{~m}, Q_{f}=0.82 \mathrm{~m}^{3} / \mathrm{s}$ and $S=0.003$} \\
\hline Run No. & $d(\mathrm{~m})$ & $d / D$ & $V_{\text {mean }}(\mathrm{m} / \mathrm{s})$ & Run No. & $d(\mathrm{~m})$ & $d / D$ & $V_{\text {mean }}(\mathrm{m} / \mathrm{s})$ \\
\hline 1 & 0.07 & 0.05 & 0.4116 & 21 & 0.62 & 0.44 & 0.9545 \\
\hline 2 & 0.10 & 0.07 & 0.4871 & 22 & 0.65 & 0.46 & 0.9747 \\
\hline 3 & 0.12 & 0.09 & 0.5330 & 23 & 0.67 & 0.48 & 1.0116 \\
\hline 4 & 0.15 & 0.11 & 0.5952 & 24 & 0.70 & 0.50 & 1.0248 \\
\hline 5 & 0.20 & 0.14 & 0.6114 & 25 & 0.73 & 0.52 & 1.0646 \\
\hline 6 & 0.23 & 0.16 & 0.6447 & 26 & 0.75 & 0.54 & 1.0871 \\
\hline 7 & 0.27 & 0.19 & 0.6815 & 27 & 0.79 & 0.56 & 1.1067 \\
\hline 8 & 0.30 & 0.21 & 0.7179 & 28 & 0.82 & 0.59 & 1.1302 \\
\hline 9 & 0.33 & 0.24 & 0.7442 & 29 & 0.86 & 0.61 & 1.1132 \\
\hline 10 & 0.35 & 0.25 & 0.7732 & 30 & 0.87 & 0.62 & 1.1346 \\
\hline 11 & 0.38 & 0.27 & 0.7947 & 31 & 0.90 & 0.64 & 1.1466 \\
\hline 12 & 0.40 & 0.29 & 0.8322 & 32 & 0.97 & 0.69 & 1.2032 \\
\hline 13 & 0.43 & 0.31 & 0.8481 & 33 & 1.00 & 0.71 & 1.1838 \\
\hline 14 & 0.45 & 0.32 & 0.8541 & 34 & 1.15 & 0.82 & 1.2448 \\
\hline 15 & 0.47 & 0.34 & 0.8583 & 35 & 1.20 & 0.86 & 1.3013 \\
\hline 16 & 0.50 & 0.36 & 0.8935 & 36 & 1.25 & 0.89 & 1.3153 \\
\hline 17 & 0.52 & 0.37 & 0.8975 & 37 & 1.30 & 0.93 & 1.3319 \\
\hline 18 & 0.55 & 0.39 & 0.9131 & 38 & 1.35 & 0.96 & 1.3355 \\
\hline 19 & 0.58 & 0.41 & 0.9480 & 39 & 1.39 & 0.99 & 1.3353 \\
\hline 20 & 0.60 & 0.43 & 0.9385 & & & & \\
\hline
\end{tabular}

Table (4) Measurements of Water Depths and Mean Velocities for Steel Culvert

\begin{tabular}{|c|c|c|c|c|c|c|c|}
\hline \multicolumn{4}{|c|}{$D=1.7 \mathrm{~m}, Q_{f}=3.19 \mathrm{~m}^{3} / \mathrm{s}$ and $S=0.0017$} & \multicolumn{4}{|c|}{$D=1.7 \mathrm{~m}, Q_{f}=3.19 \mathrm{~m}^{3} / \mathrm{s}$ and $S=0.0017$} \\
\hline Run No. & $d(\mathrm{~m})$ & $d / D$ & $V_{\text {mean }}(\mathrm{m} / \mathrm{s})$ & Run No. & $d(\mathrm{~m})$ & $d / D$ & $V_{\text {mean }}$ \\
\hline 1 & 0.10 & 0.06 & 0.5662 & 21 & 0.74 & 0.44 & 1.0873 \\
\hline 2 & 0.12 & 0.07 & 0.5842 & 22 & 0.78 & 0.46 & 1.1728 \\
\hline 3 & 0.14 & 0.08 & 0.6036 & 23 & 0.80 & 0.47 & 1.1870 \\
\hline 4 & 0.17 & 0.10 & 0.6353 & 24 & 0.85 & 0.50 & 1.1777 \\
\hline 5 & 0.20 & 0.12 & 0.6885 & 25 & 0.88 & 0.52 & 1.1958 \\
\hline 6 & 0.27 & 0.16 & 0.7871 & 26 & 0.92 & 0.54 & 1.2138 \\
\hline 7 & 0.30 & 0.18 & 0.8029 & 27 & 0.96 & 0.56 & 1.2140 \\
\hline 8 & 0.32 & 0.19 & 0.8180 & 28 & 1.00 & 0.59 & 1.2318 \\
\hline 9 & 0.36 & 0.21 & 0.8338 & 29 & 1.10 & 0.65 & 1.2478 \\
\hline 10 & 0.40 & 0.24 & 0.8704 & 30 & 1.15 & 0.68 & 1.2856 \\
\hline 11 & 0.43 & 0.25 & 0.8446 & 31 & 1.20 & 0.71 & 1.3416 \\
\hline 12 & 0.46 & 0.27 & 0.8703 & 32 & 1.25 & 0.74 & 1.3421 \\
\hline 13 & 0.50 & 0.29 & 0.8883 & 33 & 1.32 & 0.78 & 1.3579 \\
\hline 14 & 0.53 & 0.31 & 0.8974 & 34 & 1.38 & 0.81 & 1.3738 \\
\hline 15 & 0.55 & 0.32 & 0.9245 & 35 & 1.43 & 0.84 & 1.3950 \\
\hline 16 & 0.58 & 0.34 & 0.9618 & 36 & 1.48 & 0.87 & 1.4157 \\
\hline 17 & 0.60 & 0.35 & 0.9778 & 37 & 1.52 & 0.89 & 1.4316 \\
\hline 18 & 0.63 & 0.37 & 0.9964 & 38 & 1.55 & 0.91 & 1.3766 \\
\hline 19 & 0.65 & 0.38 & 1.0433 & 39 & 1.60 & 0.94 & 1.3961 \\
\hline 20 & 0.70 & 0.41 & 1.0799 & 40 & 1.67 & 0.98 & 1.4111 \\
\hline
\end{tabular}


Table (5) Measurements of Water Depths and Mean Velocities for Steel Culvert

\begin{tabular}{|c|c|c|c|c|c|c|c|}
\hline \multicolumn{4}{|c|}{$D=2.0 \mathrm{~m}, Q_{f}=4.75 \mathrm{~m}^{3} / \mathrm{s}$ and $S=0.00125$} & \multicolumn{4}{|c|}{$D=2.0 \mathrm{~m}, Q_{f}=4.75 \mathrm{~m}^{3} / \mathrm{s}$ and $S=0.00125$} \\
\hline Run No. & $d(\mathrm{~m})$ & $d / D$ & $V_{\text {mean }}(\mathrm{m} / \mathrm{s})$ & Run No. & $d(\mathrm{~m})$ & $d / D$ & $V_{\text {mean }}(\mathrm{m} / \mathrm{s})$ \\
\hline 1 & 0.07 & 0.04 & 0.4635 & 18 & 0.76 & 0.38 & 1.2283 \\
\hline 2 & 0.10 & 0.05 & 0.5644 & 19 & 0.83 & 0.42 & 1.3168 \\
\hline 3 & 0.12 & 0.06 & 0.6250 & 20 & 0.90 & 0.45 & 1.3283 \\
\hline 4 & 0.15 & 0.08 & 0.7056 & 21 & 0.95 & 0.48 & 1.3681 \\
\hline 5 & 0.18 & 0.09 & 0.7660 & 22 & 1.00 & 0.50 & 1.3950 \\
\hline 6 & 0.20 & 0.10 & 0.8046 & 23 & 1.10 & 0.55 & 1.4552 \\
\hline 7 & 0.22 & 0.11 & 0.8414 & 24 & 1.15 & 0.58 & 1.4306 \\
\hline 8 & 0.25 & 0.13 & 0.9052 & 25 & 1.20 & 0.60 & 1.4720 \\
\hline 9 & 0.30 & 0.15 & 0.9847 & 26 & 1.30 & 0.65 & 1.4898 \\
\hline 10 & 0.33 & 0.17 & 1.0241 & 27 & 1.40 & 0.70 & 1.4894 \\
\hline 11 & 0.35 & 0.18 & 1.0532 & 28 & 1.50 & 0.75 & 1.4921 \\
\hline 12 & 0.40 & 0.20 & 1.1086 & 29 & 1.65 & 0.83 & 1.4892 \\
\hline 13 & 0.42 & 0.21 & 1.1251 & 30 & 1.70 & 0.85 & 1.4978 \\
\hline 14 & 0.45 & 0.23 & 1.1673 & 31 & 1.80 & 0.90 & 1.4796 \\
\hline 15 & 0.50 & 0.25 & 1.2076 & 32 & 1.90 & 0.95 & 1.5140 \\
\hline 16 & 0.60 & 0.30 & 1.2700 & 33 & 1.98 & 0.99 & 1.5144 \\
\hline 17 & 0.70 & 0.35 & 1.2579 & & & & \\
\hline
\end{tabular}

\section{Mathematical Model}

From Manning's formula, Manning's roughness coefficient is a function of discharge $Q$, depth $d$, diameter $D$ and slope $S$. Velocities and depths thus collected were used to calculate Manning's roughness coefficient. To apply the Manning's equation, it is need to calculate the cross section geometric properties, cross section area $(A)$, wetted perimeter $(P)$, and hydraulic radius $(R)$.

\subsection{Geometric Parameters}

There are two materials were used in culvert pipe: concrete and steel with four different diameters $(1,1.4,1.7,2 \mathrm{~m})$ with four slopes $(0.001,0.003,0.0017$ and $0.00125)$. Geometric parameters calculated were cross section area, wetted perimeter and hydraulic radius. Geometric parameters are shown in Tables (6) to (9).

Table (6) Geometric Parameters for Concrete Culvert with 1.0m Diameter

\begin{tabular}{|c|c|c|c|c|c|c|c|c|c|}
\hline No. of Sec. & $d(\mathrm{~m})$ & $A\left(\mathrm{~m}^{2}\right)$ & $P(\mathrm{~m})$ & $R(\mathrm{~m})$ & No. of Sec. & $d(\mathrm{~m})$ & $A\left(\mathrm{~m}^{2}\right)$ & $P(\mathrm{~m})$ & $R(\mathrm{~m})$ \\
\hline 1 & 0.05 & 0.0147 & 0.4510 & 0.0326 & 17 & 0.50 & 0.3927 & 1.5708 & 0.2500 \\
\hline 2 & 0.10 & 0.0409 & 0.6435 & 0.0635 & 18 & 0.53 & 0.4227 & 1.6308 & 0.2592 \\
\hline 3 & 0.12 & 0.0534 & 0.7075 & 0.0755 & 19 & 0.55 & 0.4426 & 1.6710 & 0.2649 \\
\hline 4 & 0.15 & 0.0739 & 0.7954 & 0.0929 & 20 & 0.57 & 0.4625 & 1.7113 & 0.2703 \\
\hline 5 & 0.20 & 0.1118 & 0.9273 & 0.1206 & 21 & 0.60 & 0.4920 & 1.7722 & 0.2776 \\
\hline 6 & 0.22 & 0.1281 & 0.9764 & 0.1312 & 22 & 0.63 & 0.5212 & 1.8338 & 0.2842 \\
\hline 7 & 0.25 & 0.1535 & 1.0472 & 0.1466 & 23 & 0.65 & 0.5404 & 1.8755 & 0.2881 \\
\hline 8 & 0.28 & 0.1800 & 1.1152 & 0.1614 & 24 & 0.67 & 0.5594 & 1.9177 & 0.2917 \\
\hline 9 & 0.30 & 0.1982 & 1.1593 & 0.1709 & 25 & 0.70 & 0.5872 & 1.9823 & 0.2962 \\
\hline 10 & 0.33 & 0.2260 & 1.2239 & 0.1847 & 26 & 0.74 & 0.6231 & 2.0715 & 0.3008 \\
\hline 11 & 0.35 & 0.2450 & 1.2661 & 0.1935 & 27 & 0.76 & 0.6405 & 2.1176 & 0.3024 \\
\hline 12 & 0.37 & 0.2642 & 1.3078 & 0.2020 & 28 & 0.80 & 0.6736 & 2.2143 & 0.3042 \\
\hline 13 & 0.40 & 0.2934 & 1.3694 & 0.2142 & 29 & 0.86 & 0.7186 & 2.3746 & 0.3026 \\
\hline 14 & 0.42 & 0.3130 & 1.4101 & 0.2220 & 30 & 0.90 & 0.7445 & 2.4981 & 0.2980 \\
\hline 15 & 0.45 & 0.3428 & 1.4706 & 0.2331 & 31 & 0.97 & 0.7785 & 2.7934 & 0.2787 \\
\hline 16 & 0.47 & 0.3627 & 1.5108 & 0.2401 & & & & & \\
\hline
\end{tabular}


Table (7) Geometric Parameters for Concrete Culvert with 1.4m Diameter

\begin{tabular}{|c|c|c|c|c|c|c|c|c|c|}
\hline No. of Sec. & $d(\mathrm{~m})$ & $A\left(\mathrm{~m}^{2}\right)$ & $P(\mathrm{~m})$ & $R(\mathrm{~m})$ & No. of Sec. & $d(\mathrm{~m})$ & $A\left(\mathrm{~m}^{2}\right)$ & $P(\mathrm{~m})$ & $R(\mathrm{~m})$ \\
\hline 1 & 0.07 & 0.029 & 0.631 & 0.046 & 21 & 0.62 & 0.658 & 2.039 & 0.323 \\
\hline 2 & 0.10 & 0.049 & 0.758 & 0.064 & 22 & 0.65 & 0.700 & 2.099 & 0.333 \\
\hline 3 & 0.12 & 0.064 & 0.832 & 0.077 & 23 & 0.67 & 0.728 & 2.139 & 0.340 \\
\hline 4 & 0.17 & 0.106 & 0.997 & 0.107 & 24 & 0.70 & 0.770 & 2.199 & 0.350 \\
\hline 5 & 0.20 & 0.135 & 1.085 & 0.124 & 25 & 0.73 & 0.812 & 2.259 & 0.359 \\
\hline 6 & 0.23 & 0.165 & 1.169 & 0.141 & 26 & 0.75 & 0.840 & 2.299 & 0.365 \\
\hline 7 & 0.27 & 0.203 & 1.260 & 0.161 & 27 & 0.79 & 0.895 & 2.380 & 0.376 \\
\hline 8 & 0.30 & 0.242 & 1.348 & 0.179 & 28 & 0.82 & 0.937 & 2.440 & 0.384 \\
\hline 9 & 0.33 & 0.277 & 1.419 & 0.195 & 29 & 0.86 & 0.992 & 2.522 & 0.393 \\
\hline 10 & 0.35 & 0.301 & 1.466 & 0.205 & 30 & 0.87 & 1.005 & 2.543 & 0.395 \\
\hline 11 & 0.38 & 0.338 & 1.534 & 0.220 & 31 & 0.90 & 1.046 & 2.605 & 0.402 \\
\hline 12 & 0.40 & 0.363 & 1.579 & 0.230 & 32 & 0.97 & 1.138 & 2.753 & 0.413 \\
\hline 13 & 0.43 & 0.401 & 1.645 & 0.244 & 33 & 1.00 & 1.176 & 2.819 & 0.417 \\
\hline 14 & 0.45 & 0.427 & 1.688 & 0.253 & 34 & 1.15 & 1.353 & 3.177 & 0.426 \\
\hline 15 & 0.47 & 0.454 & 1.730 & 0.262 & 35 & 1.20 & 1.404 & 3.313 & 0.424 \\
\hline 16 & 0.50 & 0.494 & 1.793 & 0.275 & 36 & 1.25 & 1.451 & 3.465 & 0.419 \\
\hline 17 & 0.52 & 0.520 & 1.835 & 0.284 & 37 & 1.30 & 1.491 & 3.641 & 0.409 \\
\hline 18 & 0.55 & 0.561 & 1.897 & 0.296 & 38 & 1.35 & 1.522 & 3.866 & 0.394 \\
\hline 19 & 0.58 & 0.603 & 1.958 & 0.308 & 39 & 1.39 & 1.538 & 4.161 & 0.370 \\
\hline 20 & 0.60 & 0.630 & 1.998 & 0.315 & & & & & \\
\hline
\end{tabular}

Table (8) Geometric Parameters for Steel Culvert with 1.7m Diameter

\begin{tabular}{|c|c|c|c|c|c|c|c|c|c|}
\hline $\begin{array}{l}\text { No. of } \\
\text { Sec. }\end{array}$ & $d(\mathbf{m})$ & $A\left(\mathbf{m}^{2}\right)$ & $P(\mathbf{m})$ & $\boldsymbol{R}(\mathbf{m})$ & No. of Sec. & $d(\mathbf{m})$ & $A\left(\mathbf{m}^{2}\right)$ & $P(\mathbf{m})$ & $R(\mathbf{m})$ \\
\hline 1 & 0.10 & 0.054 & 0.833 & 0.065 & 21 & 0.74 & 0.948 & 2.450 & 0.387 \\
\hline 2 & 0.12 & 0.071 & 0.914 & 0.077 & 22 & 0.78 & 1.016 & 2.530 & 0.402 \\
\hline 3 & 0.14 & 0.089 & 0.990 & 0.090 & 23 & 0.80 & 1.050 & 2.570 & 0.408 \\
\hline 4 & 0.17 & 0.118 & 1.094 & 0.108 & 24 & 0.85 & 1.135 & 2.670 & 0.425 \\
\hline 5 & 0.20 & 0.150 & 1.190 & 0.126 & 25 & 0.88 & 1.186 & 2.730 & 0.434 \\
\hline 6 & 0.27 & 0.232 & 1.394 & 0.166 & 26 & 0.92 & 1.254 & 2.811 & 0.446 \\
\hline 7 & 0.30 & 0.270 & 1.474 & 0.183 & 27 & 0.96 & 1.321 & 2.891 & 0.457 \\
\hline 8 & 0.32 & 0.296 & 1.526 & 0.194 & 28 & 1.00 & 1.389 & 2.972 & 0.467 \\
\hline 9 & 0.36 & 0.351 & 1.626 & 0.216 & 29 & 1.10 & 1.554 & 3.178 & 0.489 \\
\hline 10 & 0.40 & 0.407 & 1.722 & 0.237 & 30 & 1.15 & 1.634 & 3.284 & 0.498 \\
\hline 11 & 0.43 & 0.451 & 1.792 & 0.252 & 31 & 1.20 & 1.713 & 3.392 & 0.505 \\
\hline 12 & 0.46 & 0.496 & 1.860 & 0.267 & 32 & 1.25 & 1.789 & 3.503 & 0.511 \\
\hline 13 & 0.50 & 0.557 & 1.949 & 0.286 & 33 & 1.32 & 1.891 & 3.666 & 0.516 \\
\hline 14 & 0.53 & 0.604 & 2.014 & 0.300 & 34 & 1.38 & 1.974 & 3.815 & 0.517 \\
\hline 15 & 0.55 & 0.636 & 2.057 & 0.309 & 35 & 1.43 & 2.038 & 3.947 & 0.516 \\
\hline 16 & 0.58 & 0.684 & 2.121 & 0.322 & 36 & 1.48 & 2.098 & 4.090 & 0.513 \\
\hline 17 & 0.60 & 0.716 & 2.163 & 0.331 & 37 & 1.52 & 2.141 & 4.214 & 0.508 \\
\hline 18 & 0.63 & 0.765 & 2.225 & 0.344 & 38 & 1.55 & 2.172 & 4.315 & 0.503 \\
\hline 19 & 0.65 & 0.798 & 2.267 & 0.352 & 39 & 1.60 & 2.216 & 4.508 & 0.492 \\
\hline 20 & 0.70 & 0.881 & 2.369 & 0.372 & 40 & 1.67 & 2.261 & 4.888 & 0.463 \\
\hline
\end{tabular}


Table (9) Geometric Parameters for Steel Culvert with 2.0m Diameter

\begin{tabular}{|c|c|c|c|c|c|c|c|c|c|}
\hline No. of Sec. & $d(\mathrm{~m})$ & $A\left(\mathrm{~m}^{2}\right)$ & $P(\mathrm{~m})$ & $R(\mathrm{~m})$ & No. of Sec. & $d(\mathrm{~m})$ & $A\left(\mathrm{~m}^{2}\right)$ & $P(\mathrm{~m})$ & $R(\mathrm{~m})$ \\
\hline 1 & 0.07 & 0.035 & 0.753 & 0.046 & 18 & 0.76 & 1.095 & 2.657 & 0.412 \\
\hline 2 & 0.10 & 0.059 & 0.902 & 0.065 & 19 & 0.83 & 1.232 & 2.800 & 0.440 \\
\hline 3 & 0.12 & 0.077 & 0.990 & 0.078 & 20 & 0.90 & 1.371 & 2.941 & 0.466 \\
\hline 4 & 0.15 & 0.107 & 1.110 & 0.096 & 21 & 0.95 & 1.471 & 3.042 & 0.484 \\
\hline 5 & 0.18 & 0.140 & 1.219 & 0.115 & 22 & 1.00 & 1.571 & 3.142 & 0.500 \\
\hline 6 & 0.20 & 0.164 & 1.287 & 0.127 & 23 & 1.10 & 1.770 & 3.342 & 0.530 \\
\hline 7 & 0.22 & 0.188 & 1.352 & 0.139 & 24 & 1.15 & 1.870 & 3.443 & 0.543 \\
\hline 8 & 0.25 & 0.227 & 1.445 & 0.157 & 25 & 1.20 & 1.968 & 3.544 & 0.555 \\
\hline 9 & 0.30 & 0.295 & 1.591 & 0.186 & 26 & 1.30 & 2.162 & 3.751 & 0.576 \\
\hline 10 & 0.33 & 0.339 & 1.673 & 0.203 & 27 & 1.40 & 2.349 & 3.965 & 0.592 \\
\hline 11 & 0.35 & 0.369 & 1.726 & 0.214 & 28 & 1.50 & 2.527 & 4.189 & 0.603 \\
\hline 12 & 0.40 & 0.447 & 1.855 & 0.241 & 29 & 1.65 & 2.772 & 4.557 & 0.608 \\
\hline 13 & 0.42 & 0.480 & 1.904 & 0.252 & 30 & 1.70 & 2.846 & 4.692 & 0.607 \\
\hline 14 & 0.45 & 0.529 & 1.977 & 0.268 & 31 & 1.80 & 2.978 & 4.996 & 0.596 \\
\hline 15 & 0.50 & 0.614 & 2.094 & 0.293 & 32 & 1.90 & 3.083 & 5.381 & 0.573 \\
\hline 16 & 0.60 & 0.793 & 2.319 & 0.342 & 33 & 1.98 & 3.136 & 5.883 & 0.533 \\
\hline 17 & 0.70 & 0.980 & 2.532 & 0.387 & & & & & \\
\hline
\end{tabular}

\subsection{Manning Roughness}

\section{Coefficient}

Manning's roughness coefficient can be calculated from Manning's equation, Eq. (1) and for full flow as:

$n_{f}=\frac{(D / 4)^{2 / 3} S^{1 / 2}}{V_{f}}$

With the four different values of bed slope obtained from four different four culverts and the water depths. Manning's roughness coefficient $n$ for each discharge in each of the four tested culverts was calculated. The calculated Manning roughness's for the four culverts are presented in Table (10).

Table (10) Values of Manning's Roughness Coefficient for Steel and Concrete Culverts Flowing Partly Full

\begin{tabular}{|l|l|l|}
\hline \multirow{2}{*}{$n$} & \multicolumn{2}{|l|}{ Concrete Culvert } \\
\cline { 2 - 3 } & $D=1.0 \mathrm{~m}$ & $D=1.4 \mathrm{~m}$ \\
\hline$n_{\min }$ & 0.0107 & 0.0169 \\
\hline$n_{\max }$ & 0.0155 & 0.0270 \\
\hline$n_{f}$ & 0.0128 & 0.0221 \\
\hline \multirow{2}{*}{$n$} & Steel Culvert \\
\cline { 2 - 3 } & $D=1.7 \mathrm{~m}$ & $D=1.7 \mathrm{~m}$ \\
\hline$n_{\min }$ & 0.0117 & 0.0117 \\
\hline$n_{\max }$ & 0.0206 & 0.0206 \\
\hline$n_{f}$ & 0.0174 & 0.0174 \\
\hline
\end{tabular}

The obtained values are in a good agreement with the values recommended by Chow [1]. The concrete surface was somewhat very smooth, as the value implied that the roughness. The concrete reference Manning's coefficient $n$ values was in between 0.011 and 0.022 , the smoothest surface finishing tested herein. It was very evident as the finishing surface of the culvert became rougher, the $n$ value increased. The largest $n$ value was 0.0270 and belonged to the concrete culvert. This value was even larger than the standard values of concrete.

\subsection{Equations of Relative Manning Coefficient with Relative Water Depth for Circular Open Channels}

In 1998, Zaghloul [13] showed that the roughness value $\mathrm{n}$ for a pipe partially full is greater than the roughness value $n_{f}$ for full flow conditions. The variation of the relative roughness with the relative depth by Zaghloul is:

$$
\begin{aligned}
& \frac{n}{n_{f}}=0.9987+3.4616(d / D)-14.7108(d / D)^{2} \\
& \quad+27.2574(d / D)^{3}-23.4963(d / D)^{4}+7.4909(d / D)^{5}
\end{aligned}
$$

In (2004), Akgiray [15] found that Camp's curve was well represented by the following equation: 
$\frac{n}{n_{f}}=1-0.8627 X^{5}+0.4281 X^{4}+0.7626 X^{3}$

$$
-1.02 X^{2}+0.8057 X
$$

Where $X=1-d / D$

In (2012), Devkota [6] showed that a linear trend represents the relation between the relative Manning's roughness coefficient and relative depth, as:

$$
\begin{aligned}
\frac{n}{n_{f}}= & 46.821(d / D)^{5}-121.61(d / D)^{4}+119.76(d / D)^{3} \\
& -56.528(d / D)^{2}+12.458(d / D)
\end{aligned}
$$

In 2012, Toews and Clark [20] divided the equation into two parts:

$$
\begin{array}{ll}
n / n_{f}=0.878(d / D)^{-0.175} & d / D \leq 0.5 \\
n / n_{f}=1 & d / D>0.5
\end{array}
$$

In 2012, Bengtson [21] developed equations for $n / n_{f}$ as a function of $d / D$, over the range from $0<d / D<1$, as:

$$
\begin{array}{lr}
n / n_{f}=1+(d / D) /(0.3) & 0 \leq d / D \leq 0.03 \\
n / n_{f}=1.1+(d / D-0.03)(12 / 7) & 0.03 \leq d / D \leq 0.1 \\
n / n_{f}=1.22+(d / D-0.1)(0.6) & 0.1 \leq d / D \leq 0.2 \\
n / n_{f}=1.29 & 0.2 \leq d / D \leq 0.3 \\
n / n_{f}=1.29-(d / D-0.3)(0.2) & 0.3 \leq d / D \leq 0.5 \\
n / n_{f}=1.25-(d / D-0.5)(0.5) & 0.5 \leq d / D \leq 1
\end{array}
$$

\section{Results and Analysis}

One of the most difficulties with open channel flow is variations in roughness of cross section. Manning's roughness coefficient is widely used and extremely important parameter for use in water flow computation including flow velocity and depth. The Manning's roughness coefficient also is affected by the relative depth. Relative depth is the ratio of depth to diameter $(d / D)$. The relative Manning's roughness is the term used to define the ratio of Manning's roughness for partial flow to full flow $\left(n / n_{f}\right)$. The goal of this study is to investigate the effect of the variation of water depth in the different types of culvert materials and to determination a relation between Manning's roughness coefficient and relative depth. The water depth profile was measured within culverts with varying discharge, slope and diameter. Discharge and depth thus collected were used to calculate Manning's roughness coefficient. The coefficients were then compared to the full flow roughness reported for steel and concrete culverts.

\subsection{Manning Roughness Coefficient with Relative Depth}

For the concrete culverts $1.0 \mathrm{~m}$ and $1.4 \mathrm{~m}$ diameter, Manning's roughness coefficients were calculated, Figures (2) to (5). Similarly for the steel culvert $1.7 \mathrm{~m}$ and $2.0 \mathrm{~m}$ diameter, Manning's roughness coefficients were calculated.

For the four tested culverts, Manning's roughness coefficients were calculated, Figures (2) to (5). From Figure (2), the values of the Manning's roughness coefficient for the concrete culvert $1.0 \mathrm{~m}$ diameter varied from 0.0107 to 0.0155 for relative depths of $5 \%$ to $97 \%$. The maximum Manning's roughness coefficient occurred at a relative depth of $70 \%$. The discharge range is from 0.0044 to $0.82 \mathrm{~m}^{3} / \mathrm{sec}$ at slope 0.001 . Similarly, the value of the Manning's Roughness Coefficient for the concrete culvert $1.4 \mathrm{~m}$ diameter varied from 0.0169 to 0.0270 for relative depths of $5 \%$ to $99.3 \%$, Figure (3). The maximum Manning's roughness coefficient occurred at a relative depth of $42.9 \%$. The discharge range is from 0.0118 to $2.053 \mathrm{~m}^{3} / \mathrm{sec}$ and slope 0.003 . The Manning's roughness value for the steel culvert $1.7 \mathrm{~m}$ diameter ranged between 0.0117 and 0.0206 for relative depths of $5.8 \%$ to $98.2 \%$, Figure (4). The maximum Manning's roughness coefficient was observed at a relative depth of $31 \%$. The Manning's roughness value for the steel culvert $2.0 \mathrm{~m}$ diameter ranged between 0.0098 and 0.017 for relative depths of $3.5 \%$ to $99 \%$, Figure (5). The maximum Manning's roughness coefficient occurred at a relative depth of $82.5 \%$. With these results, the Manning's roughness coefficient varied with diameter and water depth. 
Mansoura Engineering Journal, (MEJ), Vol. 40, Issue 2, June 201548C:

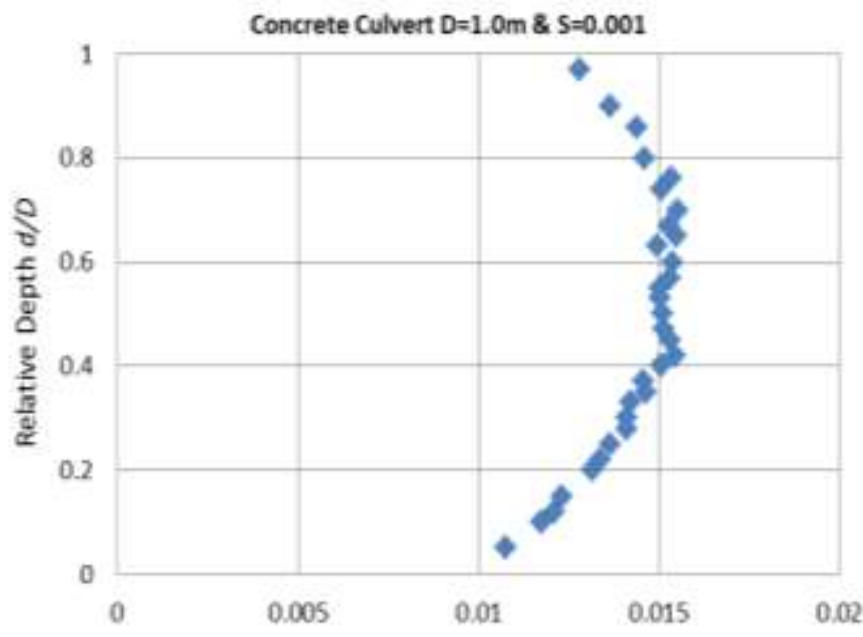

Manning's Roughness Coefficient $n$

Figure (2) Manning's Roughness versus Relative Depth in Concrete Culvert $D=1.0 \mathrm{~m}$

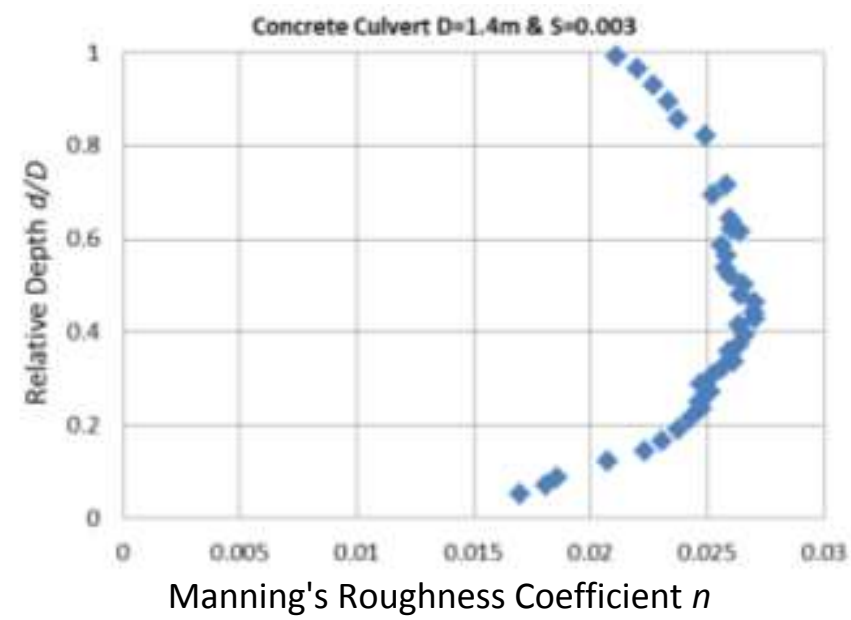

Figure (3) Manning's Roughness versus Relative Depth in Concrete Culvert $D=1.4 \mathrm{~m}$

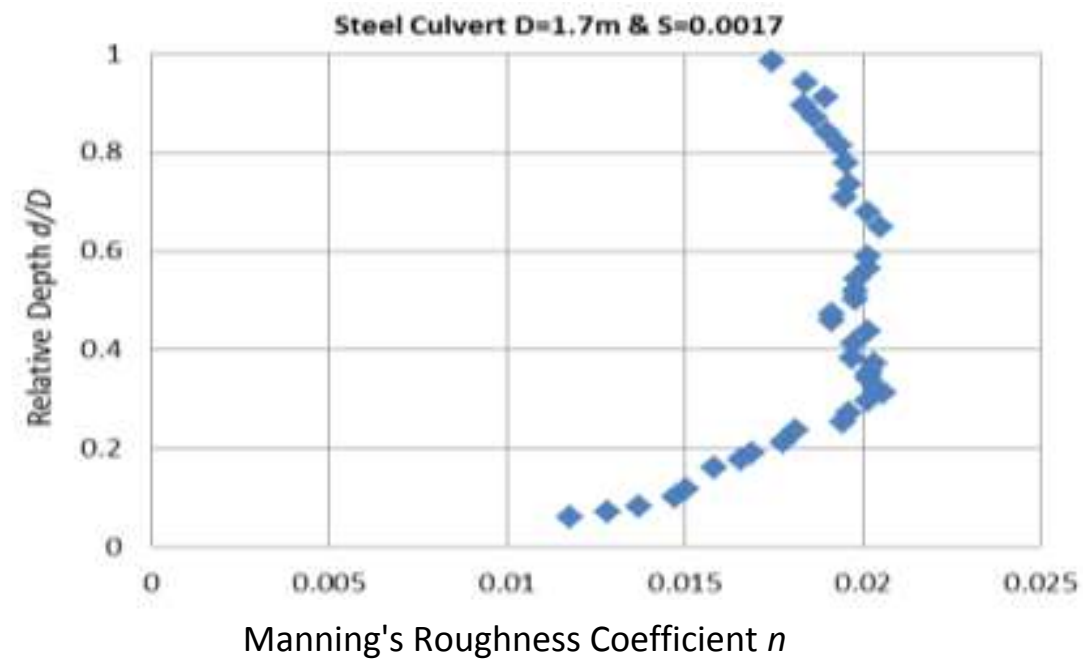

Figure (4) Manning's Roughness versus Kelatıve Depth in Steel Culvert the $D=1.7 \mathrm{~m}$ 


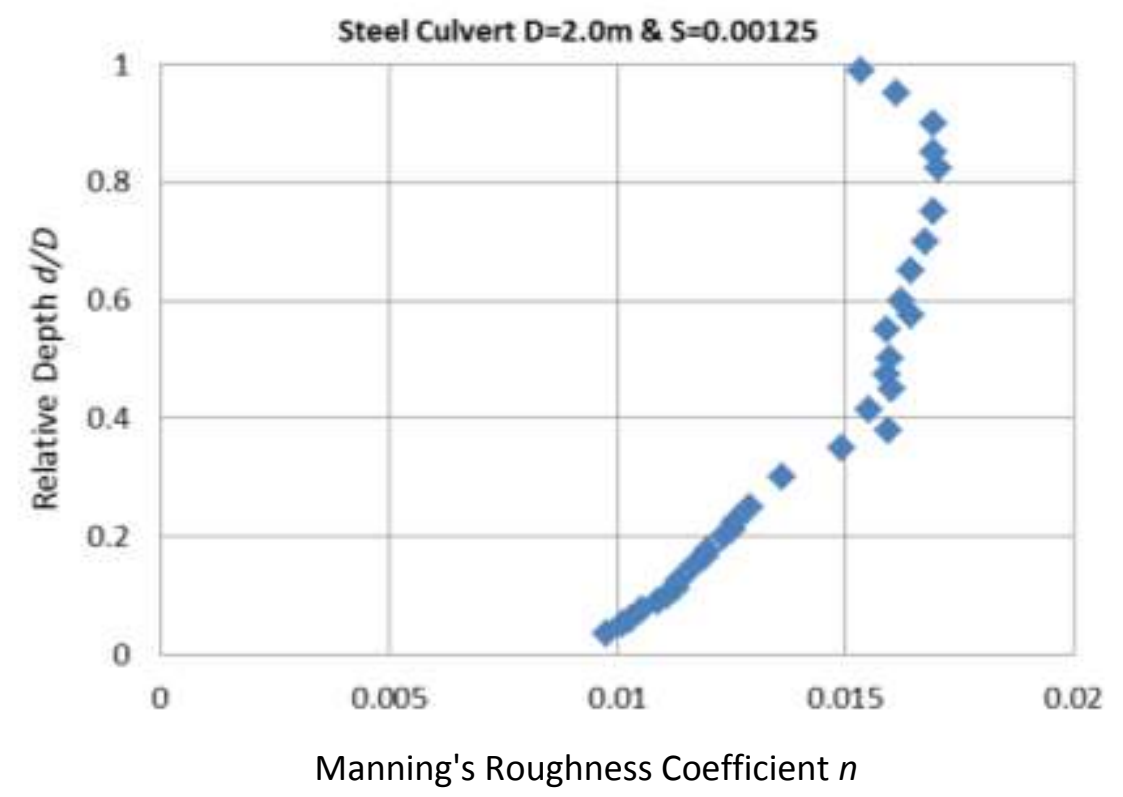

Figure (5) Manning's Roughness versus Relative Depth in Steel Culvert the $D=2.0 \mathrm{~m}$

\subsection{Relative Manning} Roughness Coefficient with Relative Depth

A plot of relative Manning's roughness versus relative water depth for four tested culverts is shown in Fig. (6). From curve, for below $25 \%$ full, the relative roughness for steel culvert is less than relative roughness for the concrete culvert and the Manning's roughness coefficient $n$ increased with the relative water depth $(d / D)$ increased. Below $25 \%$ full, the Manning's roughness coefficient $n$ increased with the relative water depth $(d / D)$ increase.

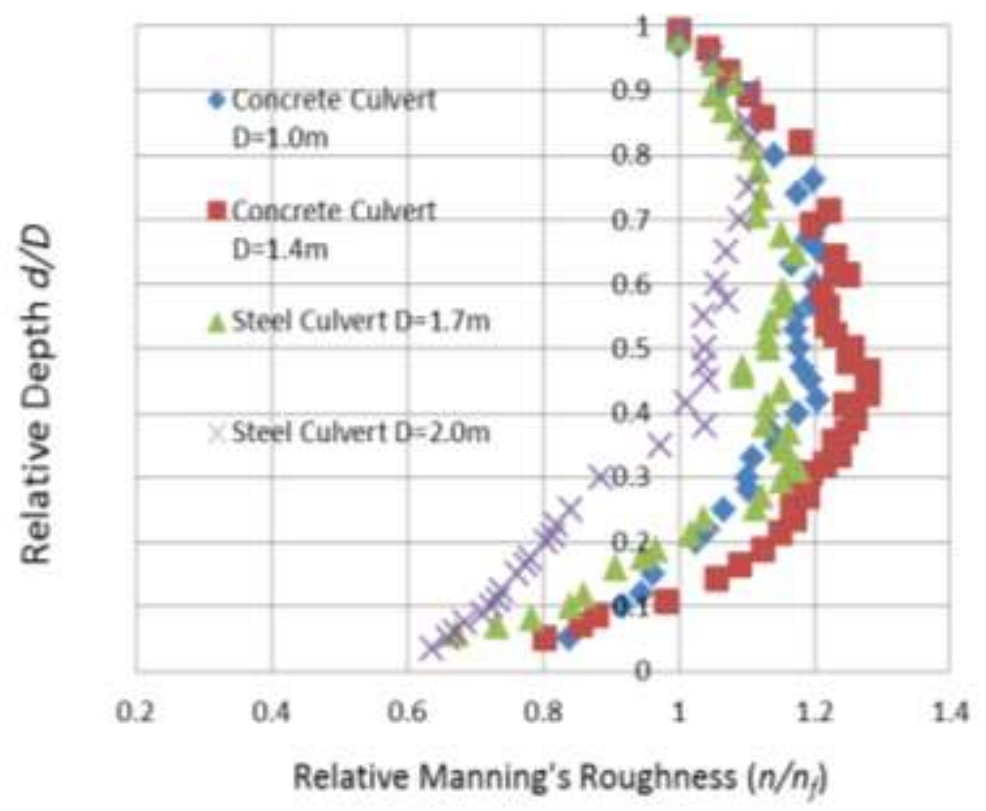

Figure (6) Relative Manning's Roughness versus Relative Depth in Four Test Culverts 
A plot of relative Manning's roughness coefficient versus relative depth for concrete culvert $1.0 \mathrm{~m}$ and $1.4 \mathrm{~m}$ diameter are shown in Fig. (6). Manning's roughness coefficient increases with the increase in diameter of the culvert. From curves, for below $10 \%$ relative depth the relative Manning's roughness value is from 80 to $100 \%$. The results presented that the value of the Manning's roughness coefficient for the steel culvert varied from 0.0098 to 0.0206 .
A reversed plot of the data with the trend line is shown in Figure (7) for relative depth less than $25 \%$. The axis of the plot of relative Manning's roughness coefficient versus relative depth was reversed to determine a trend line that shows the relation between the variations of relative Manning's roughness coefficient with relative depth in partially filled concrete and steel culverts.

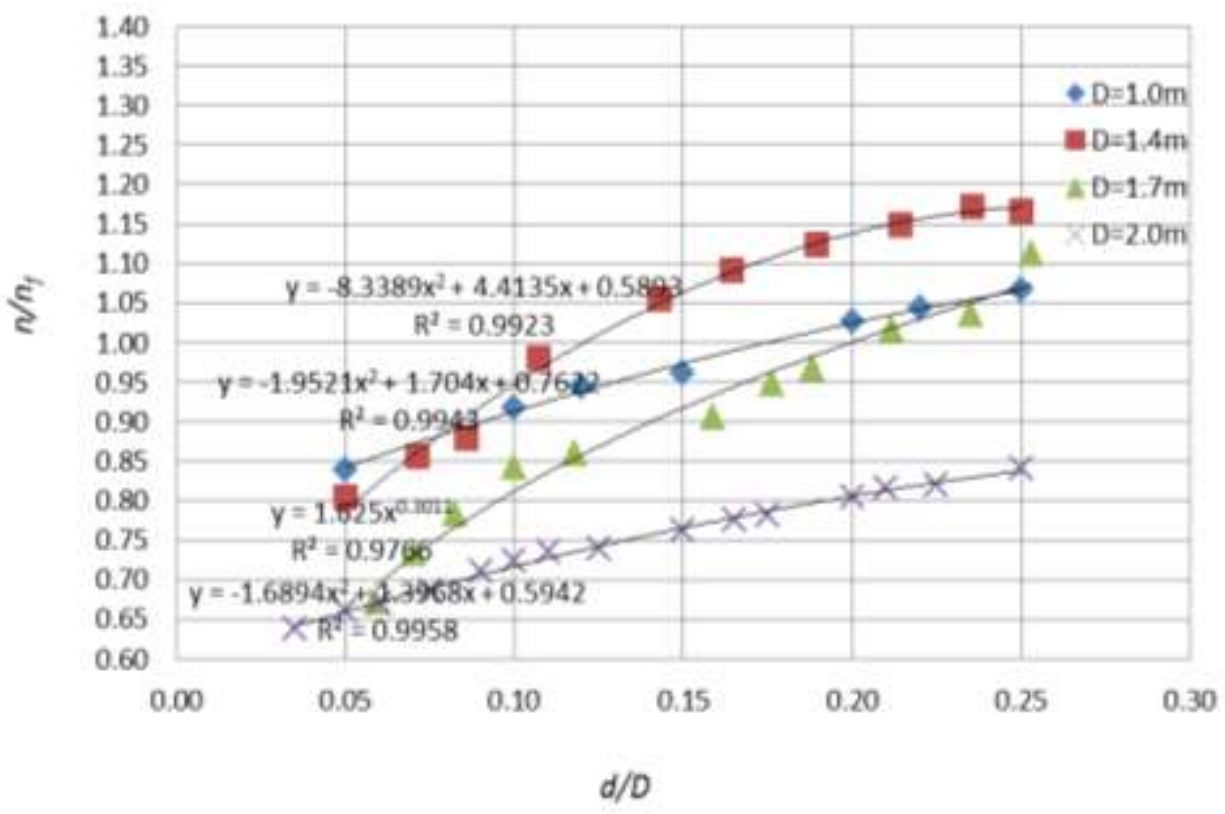

Figure (7) Trend line representing the Relation between Relative Manning's Roughness Coefficient and Relative depth in Four Test Culverts for $(d / D \leq 0.25)$

The equations for the variation of relative Manning's roughness coefficient for four tested culverts are shown below:

For concrete culvert with diameter $1.0 \mathrm{~m}$, as:

$$
n / n_{f}=-1.9521(d / D)^{2}+1.704(d / D)+0.7622
$$

For concrete culvert with diameter $1.4 \mathrm{~m}$, as:

$$
n / n_{f}=-8.3389(d / D)^{2}+4.4135(d / D)+0.5893
$$

For steel culvert with diameter $1.7 \mathrm{~m}$, as: $n / n_{f}=1.625(d / D)^{0.3011}$

For steel culvert with diameter $2.0 \mathrm{~m}$, as:

$$
n / n_{f}=-1.6894(d / D)^{2}+1.3968(d / D)+0.5942
$$

Tables (11) to (14) were presented comparison between previous equations to calculate relative Manning's roughness coefficient with proposed equations (Equations (14) to (17). From these tables, it was cleared that the proposed equations were more accurate than any previous equations. For Eq. (14) and (15) the maximum percentage error is 1.2 and $1.8 \%$,respectively. For Eq. (16) and (17) the maximum percentage error is 3.5 and $1.0 \%$ respectively. The most accurate previous equation is Devkota's equation. 
Table (11) Presents a Comparison between the Results of Field Work with Zaghloul, Akgiray, Devkota, Toews and Clark and Bengtson for Relative Manning Roughness $n / n_{f}$ in Concrete Culvert 1.0m Diameter

\begin{tabular}{|l|l|l|l|l|l|l|l|l|l|l|l|l|l|}
\hline $\begin{array}{l}\text { d/D } \\
\text { act }\end{array}$ & $\begin{array}{l}n / n_{f} \\
\text { act }\end{array}$ & Zaghloul & \%Error & Akgiray & \%Error & Devkota & \%Error & $\begin{array}{l}\text { Toews and } \\
\text { Clark }\end{array}$ & \%Error & Bengtson & \%Error & Eq. (14) & \%Error \\
\hline 0.05 & 0.840 & 1.138 & -35.5 & 1.180 & -40.5 & $\mathbf{0 . 4 9 6}$ & 40.9 & 1.483 & -76.6 & 1.167 & -38.9 & 0.843 & -0.3 \\
\hline 0.10 & 0.918 & 1.223 & -33.2 & 1.226 & -33.6 & 0.789 & 14.1 & 1.314 & -43.1 & 1.220 & -32.9 & 0.913 & 0.5 \\
\hline 0.12 & 0.945 & 1.245 & -31.7 & 1.240 & -31.3 & 0.864 & $\mathbf{8 . 6}$ & 1.272 & -34.7 & 1.232 & -30.4 & 0.939 & 0.7 \\
\hline 0.15 & 0.962 & 1.268 & -31.7 & 1.257 & -30.6 & 0.943 & 2.0 & 1.224 & -27.2 & 1.250 & -29.9 & 0.974 & -1.2 \\
\hline 0.20 & 1.027 & 1.285 & -25.1 & 1.275 & -24.1 & 1.009 & 1.8 & 1.164 & -13.3 & 1.280 & -24.6 & 1.025 & 0.2 \\
\hline 0.22 & 1.043 & 1.287 & -23.4 & 1.279 & -22.6 & 1.020 & 2.3 & 1.144 & -9.7 & 1.290 & -23.7 & 1.043 & 0.0 \\
\hline 0.25 & 1.066 & 1.286 & -20.6 & 1.283 & -20.3 & 1.024 & 4.0 & 1.119 & -5.0 & 1.290 & -21.0 & 1.066 & 0.0 \\
\hline
\end{tabular}

Table (12) Presents a Comparison between the Results of Field Work with Zaghloul, Akgiray, Devkota, Toews and Clark and Bengtson for Relative Manning's n/nf in Concrete Culvert 1.4m Diameter

\begin{tabular}{|c|c|c|c|c|c|c|c|c|c|c|c|c|c|}
\hline $\begin{array}{l}d / D \\
\text { act }\end{array}$ & $\begin{array}{l}n / n_{f} \\
\text { act }\end{array}$ & Zaghloul & \%Error & Akgiray & \%Error & Devkota & \%Error & $\begin{array}{l}\begin{array}{l}\text { Toews and } \\
\text { Clark }\end{array} \\
\end{array}$ & $\%$ Error & Bengtson & \%Error & $\begin{array}{l}\text { Eq. } \\
\text { (15) }\end{array}$ & \%Error \\
\hline 0.05 & 0.804 & 1.138 & 41.6 & 1.180 & -46.8 & 0.496 & 38.3 & 1.483 & -84.5 & 1.134 & -41.1 & 0.789 & 1.8 \\
\hline 0.07 & 0.855 & 1.180 & -38.0 & 1.202 & -40.5 & 0.642 & 24.9 & 1.393 & -62.9 & 1.171 & -36.9 & 0.862 & -0.8 \\
\hline 0.09 & 0.879 & 1.203 & -36.9 & 1.215 & -38.2 & 0.722 & 17.9 & 1.350 & -53.6 & 1.196 & -36.0 & 0.906 & -3.1 \\
\hline 0.11 & 0.981 & 1.231 & -25.5 & 1.232 & -25.6 & 0.818 & 16.6 & 1.298 & -32.3 & 1.224 & -24.8 & 0.966 & 1.5 \\
\hline 0.14 & 1.056 & 1.263 & -19.6 & 1.253 & -18.7 & 0.928 & 12.2 & 1.234 & -16.8 & 1.246 & -17.9 & 1.050 & 0.6 \\
\hline 0.16 & 1.091 & 1.275 & -16.8 & 1.263 & -15.8 & 0.969 & 11.2 & 1.204 & -10.4 & 1.259 & -15.3 & 1.089 & 0.2 \\
\hline 0.19 & 1.125 & 1.283 & -14.1 & 1.272 & -13.1 & 1.001 & 11.0 & 1.175 & -4.5 & 1.274 & -13.2 & 1.126 & -0.1 \\
\hline 0.21 & 1.149 & 1.287 & -12.0 & 1.278 & -11.2 & 1.017 & 11.5 & 1.150 & 0.0 & 1.290 & -12.3 & 1.152 & -0.3 \\
\hline 0.24 & 1.172 & 1.287 & -9.8 & 1.282 & -9.3 & 1.023 & 12.7 & 1.131 & 3.5 & 1.290 & -10.1 & 1.166 & 0.5 \\
\hline 0.25 & 1.167 & 1.286 & -10.2 & 1.283 & -9.9 & 1.024 & 12.3 & 1.119 & 4.1 & 1.290 & -10.5 & 1.171 & -0.4 \\
\hline
\end{tabular}

Table (13) Presents a Comparison between the Results of Field Work with Zaghloul, Akgiray, Devkota, Toews and Clark and Bengtson for Relative Manning Roughness $\mathbf{n} / \mathbf{n f}$ in Steel Culvert 1.7m Diameter

\begin{tabular}{|l|l|l|l|l|l|l|l|l|l|l|l|l|l|}
\hline $\begin{array}{l}d / D \\
\text { act }\end{array}$ & $\begin{array}{l}n / n_{f} \\
\text { act }\end{array}$ & Zaghloul & \%Error & Akgiray & \%Error & Devkota & \%Error & $\begin{array}{l}\text { Toews and } \\
\text { Clark }\end{array}$ & \%Error & Bengtson & \%Error & $\begin{array}{l}\text { Eq. } \\
(16)\end{array}$ & \%Error \\
\hline 0.06 & 0.672 & 1.157 & -72.0 & 1.189 & -76.9 & 0.560 & 16.7 & 1.442 & -114.4 & 1.149 & -70.9 & 0.692 & -3.0 \\
\hline 0.07 & 0.733 & 1.179 & -60.8 & 1.201 & -63.8 & 0.637 & 13.1 & 1.396 & -90.4 & 1.170 & -59.5 & 0.731 & 0.2 \\
\hline 0.08 & 0.783 & 1.198 & -53.0 & 1.212 & -54.7 & 0.704 & 10.1 & 1.359 & -73.5 & 1.190 & -51.9 & 0.766 & 2.2 \\
\hline 0.10 & 0.842 & 1.223 & -45.2 & 1.226 & -45.6 & 0.789 & 6.3 & 1.314 & -56.0 & 1.220 & -44.9 & 0.812 & 3.5 \\
\hline 0.12 & 0.861 & 1.242 & -44.3 & 1.239 & -43.9 & 0.856 & 0.5 & 1.277 & -48.3 & 1.246 & -44.7 & 0.853 & 0.9 \\
\hline 0.16 & 0.907 & 1.272 & -40.3 & 1.261 & -39.0 & 0.960 & -5.9 & 1.212 & -33.6 & 1.259 & -38.8 & 0.934 & -3.0 \\
\hline 0.18 & 0.948 & 1.280 & -35.0 & 1.268 & -33.8 & 0.987 & -4.1 & 1.189 & -25.5 & 1.274 & -34.4 & 0.964 & -1.7 \\
\hline 0.19 & 0.967 & 1.283 & -32.7 & 1.272 & -31.5 & 1.000 & -3.4 & 1.176 & -21.6 & 1.289 & -33.2 & 0.983 & -1.6 \\
\hline 0.21 & 1.018 & 1.287 & -26.5 & 1.278 & -25.5 & 1.016 & 0.1 & 1.152 & -13.2 & 1.290 & -26.8 & 1.018 & -0.1 \\
\hline 0.24 & 1.037 & 1.287 & -24.2 & 1.281 & -23.6 & 1.023 & 1.3 & 1.131 & -9.1 & 1.290 & -24.4 & 1.051 & -1.4 \\
\hline 0.25 & 1.114 & 1.286 & -15.4 & 1.283 & -15.2 & 1.024 & 8.1 & 1.117 & -0.3 & 1.290 & -15.8 & 1.074 & 3.5 \\
\hline
\end{tabular}


Table (14) Presents a Comparison between the Results of Field Work with Zaghloul, Akgiray, Devkota, Toews and Clark and Bengtson for Relative Manning Roughness $\mathbf{n} / \mathbf{n f}$ in Steel Culvert 2.0m Diameter

\begin{tabular}{|c|c|c|c|c|c|c|c|c|c|c|c|c|c|}
\hline $\begin{array}{l}d / D \\
\text { act }\end{array}$ & $\begin{array}{l}n / n_{f} \\
\text { act }\end{array}$ & Zaghloul & \%Error & Akgiray & \%Error & Devkota & $\%$ Error & $\begin{array}{l}\text { Toews and } \\
\text { Clark } \\
\end{array}$ & $\%$ Error & Bengtson & \%Error & $\begin{array}{l}\text { Eq. } \\
(17)\end{array}$ & \%Error \\
\hline 0.04 & 0.637 & 1.103 & -73.1 & 1.162 & -82.4 & $\mathbf{0 . 3 7 2}$ & 41.6 & 1.579 & -147.8 & 1.109 & -74.0 & 0.641 & -0.6 \\
\hline 0.05 & 0.660 & 1.138 & -72.4 & 1.180 & -78.6 & 0.496 & 24.9 & 1.483 & -124.6 & 1.134 & -71.7 & 0.660 & 0.1 \\
\hline 0.06 & 0.671 & 1.159 & -72.7 & 1.191 & -77.4 & 0.568 & 15.3 & 1.437 & -114.0 & 1.151 & -71.5 & 0.672 & -0.1 \\
\hline 0.08 & 0.687 & 1.186 & -72.8 & 1.205 & -75.5 & 0.663 & 3.4 & 1.382 & -101.2 & 1.177 & -71.4 & 0.689 & -0.4 \\
\hline 0.09 & 0.711 & 1.209 & -70.2 & 1.218 & -71.4 & 0.743 & -4.6 & 1.338 & -88.3 & 1.203 & -69.3 & 0.706 & 0.6 \\
\hline 0.10 & 0.723 & 1.223 & -69.0 & 1.226 & -69.5 & 0.789 & -9.0 & 1.314 & -81.6 & 1.220 & -68.6 & 0.717 & 0.9 \\
\hline 0.11 & 0.735 & 1.234 & -68.0 & 1.234 & -67.9 & 0.829 & -12.8 & 1.292 & -75.8 & 1.226 & -66.9 & 0.727 & 1.0 \\
\hline 0.13 & 0.740 & 1.249 & -68.8 & 1.243 & -68.0 & 0.880 & -18.9 & 1.263 & -70.7 & 1.235 & -66.9 & 0.742 & -0.3 \\
\hline 0.15 & 0.762 & 1.268 & -66.5 & 1.257 & -65.1 & 0.943 & -23.9 & 1.224 & -60.7 & 1.250 & -64.1 & 0.766 & -0.6 \\
\hline 0.17 & 0.776 & 1.275 & -64.3 & 1.263 & -62.8 & 0.970 & -25.0 & 1.203 & -55.1 & 1.259 & -62.2 & 0.779 & -0.3 \\
\hline 0.18 & 0.782 & 1.279 & -63.6 & 1.267 & -62.0 & 0.985 & -25.9 & 1.191 & -52.3 & 1.265 & -61.7 & 0.787 & -0.6 \\
\hline 0.20 & 0.805 & 1.285 & -59.7 & 1.275 & -58.4 & 1.009 & -25.4 & 1.164 & -44.6 & 1.280 & -59.0 & 0.806 & -0.1 \\
\hline 0.21 & 0.816 & 1.287 & -57.6 & 1.277 & -56.4 & 1.015 & -24.4 & 1.154 & -41.3 & 1.290 & -58.0 & 0.813 & 0.4 \\
\hline 0.23 & 0.819 & 1.287 & -57.1 & 1.280 & -56.2 & 1.021 & -24.6 & 1.140 & -39.1 & 1.290 & -57.4 & 0.823 & -0.4 \\
\hline 0.25 & 0.842 & 1.286 & -52.8 & 1.283 & -52.4 & 1.024 & -21.6 & 1.119 & -32.9 & 1.290 & -53.2 & 0.838 & 0.5 \\
\hline
\end{tabular}

\section{Conclusions}

From the rustles of the presented study the following conclusions are:

1. The Manning's roughness coefficient varied with diameter and relative depth;

2. The obtained values of the Manning's roughness coefficient $n$ for sections are in a good agreement with the references values;

3. New relations between the relative depth $(d / D)$ and the relative roughness $\left(n / n_{f}\right)$ for the test culverts are presented for $d / D \leq 0.25$;

4. The new equations were compared by previous equations and it gives least error;

5. For $d / D \leq 0.25$ the partial flow roughness is less than the full flow roughness; and

6. Below $25 \%$ full, the relative roughness for steel culvert is less than relative roughness for the concrete culvert.

\section{References}

[1] Chow, V.T., "Open channel hydraulics", International student edition, McGraw-Hill Kogakusha, Ltd, 1959.
[2] Yarnell, D.L. and Woodward, S.M.1920. The flow of water in drain tile. U.S. Department of Agriculture, Bulletin 854.

[3] Camp, T.R. 1946. Design of sewers to facilitate flow. Sewage Works Journal, 18(1), 3-16.

[4] Straub L. and Morris H., "Hydraulic Tests on Corrugated Metal Culvert Pipes", University of Minnesota, 1950.

[5] Cosens, K. W., Sewer Pipe Roughness Coefficients. Sewage and Industrial Wastes 26(1), 42-50, 1954.

[6] Neale, L. C. and Price, R. E., Flow Characteristics of PVC Sewer Pipe. Journal of Sanitary Engineering Division, (Div. Proc. 90SA3), 109129, 1964.

[7] Pomeroy, R. D., Flow Velocities in Small Sewers. Journal (Water Pollution Control Federation) 39(9), 1525-1548, 1967.

[8] Barr, D.I.H., and Das, M.M., Direct solutions for normal depth using the Manning equation. Proceedings of the Institution of Civil Engineers, Part 2, 81: 315-333 1986.

[9] Saatçı, A.M., Velocity and depth of flow calculations in partially filled pipes. ASCE Journal of 
Environmental Engineering, 116(6): 1202-1212, 1990.

[10] Saatçı, A.M., Closure by author. ASCE Journal of Environmental Engineering, 118(3): 454, 1992.

[11] Esen, I.I., Design of sewers based on minimum velocity. ASCE Journal of Environmental Engineering, 119(3): 591-594, 1993.

[12] Li, K.S., Discussion of: Design of sewers based on minimum velocity by I.I. Esen. ASCE Journal of Environmental Engineering, 120(5): 1348-1350, 1994.

[13] Zaghloul, N.A., Flow Simulation in Circular Pipes with Variable Roughness using SWMM-EXTRAN Model, Journal of Hydraulic Engineering, Vol. 124, No. 1, January 1998.

[14] Giroud, J.P., Palmer, B., and Dove, J.E. 2000. Calculation of flow velocity in pipes as a function of flow rate. Geosynthetics International, 7(4-6): 583-600.

[15] Akgiray, Ö., Simple formulae for velocity, depth of flow, and slope calculations in partially filled circular pipes. Environmental Engineering Science, 21(3): 371-385, 2004.

[16] Akgiray Omer, Explicit solutions of the Manning equation for partially filled circular pipes. Canadian Journal of Civil Engineering, 32(3):490-499.

[17] American Concrete Pipe Association, "Partial Flow Condition for Culverts", 2009.

[18] Mangin S. F., "Development of an Equation Independent of Manning's Coefficient $\mathrm{n}$ for Depth Predictions in Partially-Filled Circular Culverts", MSc Thesis, Youngstown, State University, 2010.

[19] Devkota J. P., "Variation in Manning's Roughness Coefficient with Diameter", Discharge and Slope in Partially Filled HDPE Culverts, MSc Thesis, Youngstown, State University, 2012.
[20] Toews J. S. and Clark S. P., "Relative Depth Effects on Corrugated Culvert Roughness", Journal of Water Resource and Protection, (4) (838-841), 2012.

[21] Bengtson H. H., Spreadsheet Use for Partially Full Pipe Flow Calculations, Continuing Education and Development, Inc., Course No: C02-037, Credit: 2 PDH, 2012.

\section{List of notation}

A Water area;

d Water depth;

D Diameter of circular channel;

$g$ Gravitational acceleration;

Ks Absolute material roughness;

$n$ Manning's roughness coefficient;

$n_{f} \quad$ Manning's roughness coefficient for full flow;

$n_{\max } \quad$ Maximum Manning's roughness coefficient;

$n_{\min } \quad$ Minimum Manning's roughness coefficient;

$P \quad$ Wetted perimeter;

$R \quad$ Hydraulic radius;

$Q \quad$ Discharge;

$S$ Bed slope;

$V \quad$ Mean flow velocity at section;

$V_{0.2} \quad$ Flow velocity at 0.2 depth;

$V_{0.6} \quad$ Flow velocity at 0.6 depth;

$V_{0.8} \quad$ Flow velocity at 0.8 depth;

$V_{\text {mean }}$ Mean flow velocity at section;

$X$ Dimensionless variable $(X=1-h / D)$; and $y$ Vertical water depth. 